\title{
Efeitos da terapia craniossacral e a mobilização uterina no alívio da dismenorreia primária: um estudo piloto
}

\author{
Effects of cranial sacral therapy and \\ uterine mobilization on the relief of \\ primary dysmenorrhea: a pilot study
}

FisiSenectus. Unochapecó Ano 7, n. 2 - Jul/Dez. 2019 p. $12-22$

\section{Mariza Barbosa Canário. mariza_canario@hotmail.com}

Fisioterapeuta, graduada pela Faculdade de Integração do Sertão - FIS, Serra Talhada/PE.

Daphne Gilly. daphnegilly@hotmail.com

Mestre em Fisioterapia pela Universidade Federal de Pernambuco - UFPE. Docente na Faculdade de Integração do Sertão - FIS em Serra Talhada/PE. Atua nas áreas de Uroginecologia, Obstetrícia, Osteopatia e Terapia Manual.

\section{Resumo}

Introdução: a dismenorreia primária, conhecida também como cólica menstrual, é uma dor que ocorre antes ou durante o período menstrual e se caracteriza por diversos sintomas, entre eles dor no baixo ventre, cefaleia, náuseas, tontura e até desmaios. Objetivo: avaliar os efeitos da Mobilização Uterina associada ou não à Terapia Craniossacral no alívio da dismenorreia primária e seus sintomas associados. Metodologia: tratase de um estudo piloto realizado em quatro fases.A primeira consistiuna aplicação de quatro questionários (avaliativo, instrumento de rastreamento da síndrome pré-menstrual, avaliação e impacto da dismenorreia primária e cefaleia); em seguida, foi realizada a randomização de um total de oito voluntárias, quatro para o Grupo Controle (GC), que recebeu apenas a Mobilização Uterina, e quatro ao Grupo Experimental (GE), que, associada, recebeu a Terapia Craniossacral. 0 terceiro passo se caracterizou pela aplicação do questionário de satisfação, e a quarta etapa foi a entrega de um questionário de acompanhamento para que a voluntária respondesse ao fim do ciclo menstrual seguinte. Resultados: verificou-se que, no GE no qual foi aplicada a associação das duas técnicas, houve uma redução maior de sintomas associados que no GC. Conclusão: são necessários novos estudos com uma amostra maior para uma melhor análise dos resultados da aplicação dessas técnicas associadas, a fim de que sejam desenvolvidos protocolos de tratamentos fisioterapêuticos da dismenorreia primária e seus sintomas associados.

\section{Palavras-chave:}

Dismenorreia; Manipulação osteopática.

\section{Abstract}

Introduction: primary dysmenorrhea, also known as menstrual cramps, is a pain that occurs before or during the menstrual period, characterized by several symptoms, including lower abdominal pain, headache, nausea, dizziness and even fainting. Objective:to evaluate the effects of uterine mobilization associated or not with Sacral Skull Therapy in the relief of primary dysmenorrhea and its associated symptoms. Methodology: this is a four-phase pilot study: the first with the application of four questionnaires (evaluative, premenstrual

\section{Fisi enectus}


syndrome screening tool, assessment and impact of primary dysmenorrhea and headache), then, a total of eight volunteers were randomized, four to the Control Group (CG) that received only the Uterine Mobilization and four to the Experimental Group (GE) Sacral Skull Therapy. The third step is characterized by the application of the satisfaction questionnaire and the fourth step was the delivery of a follow-up questionnaire for the volunteer to respond to the end of the next menstrual cycle. Results: it was found that in the EG in which the association of the two techniques was applied, there was a greater reduction in associated symptoms than the CG. Conclusion: further studies with a larger sample are needed for a better analysis of the results of the application of these associated techniques to develop protocols for physiotherapeutic treatment of primary dysmenorrhea and its associated symptoms.

\section{Keywords:}

Dysmenorrhoea; Sacral skull therapy; Visceral mobilization.

\section{Introdução}

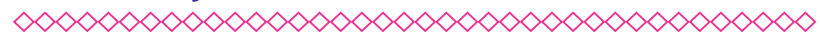

Ao longo da vida, as mulheres passam por várias etapas marcantes. Entre elas, ainda na adolescência, ocorre a menstruação, que se trata de um sangramento vaginal periódico, que tem início com a menarca - primeira menstruação espontânea, que ocorre normalmente entre 11 e 15 anos - e termina com a menopausa, que em geral ocorre entre os 45 e 50 anos, sendo a última menstruação espontânea da vida, caracterizada por, ao menos, 12 meses consecutivos de amenorreia ${ }^{1,2}$.

A síndrome pré-menstrual (SPM) é caracterizada pela ocorrência recorrente de um conjunto de alterações físicas, cognitivas, comportamentais e de humor que interferem negativamente nas atividades estudantis e laborais. Pode ter início por volta de duas semanas antes da menstruação e alívio de um a três dias após o início do fluxo menstrual ${ }^{3}$.

Vários incômodos estão presentes na síndrome pré-menstrual, no entanto a dismenorreia, mais conhecida como cólica menstrual, que ocorre no baixo ventre, é a mais comum. Sintomas como náuseas, vômitos, diarreia, cefaleia, sudorese, dor na região lombossacral e membros inferiores podem estar associados ${ }^{4}$.

A dismenorreia pode ser classificada, a partir da intensidade álgica, como leve, moderada e grave. De acordo com a etiologia, é subdividida em: primária, quando ocorre em mulheres sem nenhuma causa orgânica; e secundária, quando associada à patologia subjacente ${ }^{5}$. 0 desconforto em algumas mulheres se torna tão intenso a ponto de interromper as atividades habituais, como tarefas laborais e acadêmicas ${ }^{6}$.

Existem recursos fisioterapêuticos não invasivos alternativos para o tratamento da dismenorreia primária, tais como o Pilates ${ }^{7}$, a Acupuntura ${ }^{8}$ e a Estimulação Elétrica Nervosa Transcutânea $\left(\right.$ TENS) ${ }^{9}$.

A Osteopatia, idealizada pelo médico Dr. Andrew Taylor Still, é baseada no funcionamento das leis naturais da matéria, do movimento e da mente, desenvolvendo, assim, técnicas não invasivas usando o movimento do corpo para restabelecer sua homeostase. Esses recursos são utilizados como possíveis métodos de tratamento para a dismenorreia primária, entre eles a Terapia Craniossacral e a Mobilização Uterina ${ }^{10}$.

A Terapia Craniossacral consiste em um método suave de correção que encoraja os mecanismos naturais de cura do organismo do indivíduo a dissipar os efeitos negativos causados pela quebra da homeostase gerada pela disfunção sobre o sistema nervoso central. É uma técnica suave e não invasiva de mobilização direta do crânio e indireta do sacro que utiliza um toque com pressão inferior a cinco gramas e que pode liberar restrições no sistema craniossacral para melhorar o funcionamento do sistema nervoso central e autônomo ${ }^{11}$.

Vinda da Osteopatia Visceral, a Mobilização Uterina é uma técnica que consiste em mobilizar indiretamente o útero e consequentemente ovários através de terapia manual, com uma pressão pequena aplicada à víscera, tendo como objetivo restabelecer a mobilidade perdida. Promove, assim, o aumento da circulação, que é diminuída no 
período menstrual devido à elevação na liberação de prostaglandinas, causando a dismenorreia, e dáequilíbrio ao sistema uterino e ovariano ${ }^{12}$.

Diante do exposto, este estudo buscou avaliar os efeitos da Mobilização Uterina associada ou não à Terapia Craniossacral no alívio da dismenorreia primária e sintomas associados.

\section{Metodologia}

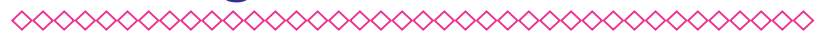

Este estudo foi realizado na Faculdade de Integração do Sertão - FIS, executado de acordo com as normas da Resolução no 466/12, da Comissão Nacional de Ética em Pesquisa/Conselho Nacional de Saúde (Conep/CNS) e a Resolução n 510/16,e aprovado pelo Comitê de Ética em Pesquisa da FIS, sob número do parecer 3.110.521.

Foram incluídas na pesquisa mulheres com idade entre 20 e 28 anos, matriculadas na instituição de ensino superior (IES), que apresentavam queixa de dismenorreia primária em seu período pré-menstrual e menstrual, sem diferenciação das fases do ciclo,e que tivessem realizado ao menos uma consulta ginecológica no último ano. Foram excluídas mulheres amenorreicas, alcoólatras, tabagistas, que possuíam alguma doença ginecológica (endometriose, síndrome dos ovários policísticos, mioma, cistos ovarianos, malformações congênitas do trato urinário e estenose cervical), além de mulheres que faziam uso de Dispositivo Intrauterino (DIU), uso de drogas analgésicas ou relaxantes musculares e que faziam qualquer outra terapia para tratamento da dismenorreia primária.

A princípio, as voluntárias foram contatadas verbalmente, com a visita da pesquisadora às salas dos cursos de Fisioterapia e Enfermagem, sendo explicados os objetivos da pesquisa e seus critérios de inclusão. As que se dispuseram a participar, apresentaram-se à pesquisadora e, então, foram conduzidas a uma sala privada na Clínica Escola de Fisioterapia (Clinefis), onde receberam esclarecimentos do estudo através da leitura do Termo de Consentimento Livre e Esclarecido (TCLE), que foi assinado pelas voluntárias que aceitaram participar.
A amostra contou com um total de oito voluntárias, divididas em dois grupos: quatro no Grupo Controle (GC), que recebeu apenas a Mobilização Uterina, e quatro no Grupo Experimental (GE), que foi submetido à associação da Mobilização Uterina à Terapia Craniossacral, sendo realizada apenas uma sessão na fase sintomática da dor, de acordo com a randomização. 0 processo de randomização foi realizado por meio de números sequenciais mantidos em envelopes opacos e retirados pela voluntária. Os números pares corresponderam ao Grupo Controle (GC), e os números ímpares, ao Grupo Experimental (GE).

Ambos os grupos foram submetidos à aplicação, pela pesquisadora, de um total de quatro questionários: no primeiro momento, foi aplicado o questionário estruturado, elaborado pelas autoras, pelo qual foram colhidos dados sociodemográficos, dados relacionados à vida cotidiana da voluntária e informações ginecológicas e obstétricas.

0 segundo questionário aplicado foi o Instrumento de Rastreamento de Sintomas Pré-Menstruais (PSST), de autoaplicação, preenchido em um único momento.Esse questionário possui validação clínica por Henz (2016) e deve ser considerado como uma ferramenta de triagem diagnóstica. As características da SPM são descritas em 19 itens, sendo 14 relacionados aos sintomas da SPM e cinco questões relacionadas às consequências desses sintomas. Para seobter o diagnóstico de SPM, é necessário que a paciente apresente ao menos um sintoma moderado a severo nos itens de 1 a 4; pelo menos quatro sintomas nos itens de 1 a 14; e ao menos um item de A-E. Ao final, o distúrbio pôde ser classificado como: ausente/nada, leve, moderado e severo ${ }^{13,14}$.

O terceiro questionário aplicado corresponde à classificação da dismenorreia.Tal questionário foi elaborado por Rodrigues et al., no ano de 2011, e é composto por 24 itens, que aferem os dados sociodemográficos, o peso e a altura,os hábitos de vida, as características do ciclo menstrual e a presença, duração, severidade, tratamento e limitações da dismenorreia ${ }^{14}$.

Como a cefaleia é um dos sintomas mais frequentes associados à dismenorreia, o quarto questionário aplicado foi o Questionário de Avaliação 
da Cefaleia, composto por dez itens, que traziam questões sobre a prevalência, característica, intensidade, tipo, duração e interferência da cefaleia na realização das atividades de vida diária ${ }^{15}$.

Após a aplicação dos quatro questionários, deu-se início à aplicação das técnicas de terapia manual. No Grupo Controle (GC), a voluntária posicionou-se em decúbito dorsal com os joelhos fletidos e foi então realizada uma pompagem sacral. Logo em seguida, a pesquisadora se posicionou ao lado da pelve da voluntária, sustentando a perna homolateral da mesma com o antebraço, enquanto apoiava a mesma mão (caudal) no joeIho contralateral, realizando então uma rotação e lateroflexão da pelve com membros inferiores. A mão cranial se posicionou em concha sobre 0 útero ( $3 \mathrm{~cm}$ acima da sínfise púbica) realizando uma leve pressão, menor que $5 \mathrm{~g}$ sobre a víscera, e efetuando ao mesmo tempo movimentos lentos e suaves de lateroversão e rotação do útero por cinco minutos, segundo o método de Jean Pierre Barral ${ }^{16}$. Essa técnica tem como objetivo normalizar as tensões faciais do útero e foi acompanhada de ventilação diafragmática de forma lenta e suave, com frenolabial, ensinada à voluntária antes da aplicação da técnica.

No Grupo Experimental (GE), a voluntária foi colocadano mesmo posicionamento do Grupo Controle (GC) e recebeu a pompagem do sacro, seguida da Mobilização Uterina. Associada à Mobilização Uterina, foi então realizada a Mobilização Craniana, em que as mãos da pesquisadora se posicionaram nos côndilos occipitais, e então feitos os seguintes movimentos: a cada duas inspirações, de forma leve e suave, o movimento de extensão da cabeça e, a cada duas expirações, realizado também o movimento lento e suave de flexão da cabeça, por cinco minutos, seguindo os princípios de William G. Sutherland ${ }^{17}$. Os movimentos de flexão e extensão são realizados apenas no nível da articulação occipito-atlanto-axial, respeitando-se sua amplitude de movimento sem que entrem movimentos de flexão e extensão de pescoço da participante.

Em ambos os grupos, as técnicas foram aplicadas no momento em que a voluntária relatou dismenorreia. Após a aplicação no Grupo Controle ou Grupo Experimental, a voluntária repousou por mais cinco minutos em decúbito dorsal. Logo em seguida, foi posicionada cuidadosamente sentada, com a ajuda da pesquisadora, sendo então aplicado questionário estruturado de satisfação elaborado pelas autoras sobre a aplicação das técnicas. A paciente relatou se houve ou não o alívio da dismenorreia primária e os sintomas associados, como: cefaleia, náuseas, dor na região lombossacral e membros inferiores.0 questionário foi aplicado nos dois grupos da mesma maneira.

Foi entregue, então, questionário elaborado pela autora para follow up, ou seja, acompanhamento da voluntária no próximo ciclo menstrual, em queesta responde em uma escala de 0 a 10, correspondendo 0 a nenhuma dor, o que significa uma melhora completa dos sintomas, e 10 uma dor severa, não ocorrendo nenhuma melhora. Os sintomas abordados foram: dor em baixo-ventre, cefaleia, náuseas, dor na virilha, dor na região lombossacral e dor em membros inferiores.

Para análise dos dados, foram realizadosa tabulação dos resultados no softwareestatístico SPSS Statistics (20.0) e o teste t de student pareado para análise da variância da condição pré e pós-tratamento. Os resultados foram descritos em tabelas.

\section{Resultados}

$\infty<\infty<\infty<\infty<\infty<\infty<\infty<\infty<\infty<\infty<\infty<\infty<\infty<\infty<\infty<\infty$

A amostra inicial contou com 30 voluntárias, porém, ao ser realizada a avaliação, foram excluídas 22 mulheres, pois 12 não haviam realizado consulta ginecológica há um ano e 10 possuíam a síndrome dos ovários policísticos, resultando em uma população de 8 participantes distribuídas em dois grupos: 4 (50\%) no Grupo Controle (GC) e 4 (50\%) no Grupo Experimental (GE). Segundo o teste $t$ de student pareado, ambos os grupos obtiveram significância da variância no pré e pós-tratamento: no GC p de 0,015 e no GE p de 0,014, sendo que em ambos os grupos houve diferença, $p<0,05$. A média de idade do GC foi de $23( \pm 2,16)$ anos e de $22,25( \pm 1,7)$ anos no GE (Tabela $\mathbf{1})$.

Segundo o Instrumento de Rastreamento de Sintomas Pré-Menstruais (PSST), 6 (75\%) das 8 voluntárias que participaram do estudo, sendo 2 do $\mathrm{GC}$ e 4 do GE, obtiveram diagnóstico da Síndrome 
Pré-Menstrual de acordo com os sintomas, consequências e interferência em sua vida. As principais variáveis avaliadas a partir do questionário de dismenorreia são apresentados a seguir.

Em relação à classificação da quantidade de sangue perdida, $1(n=2)$ do $G C$ respondeu que é abundante e $(n=3)$ do $G E$ responderam ser normal. Em ambos os grupos, as voluntárias relataram que o ciclo menstrual é regular e que sentiram dor na parte inferior do abdômen nos últimos seis meses. No $G C,(n=3)$ e todas do $G E$ relataram que têm dor desde a primeira menstruação. A média de intensidade da dor foi moderada em $(n=3)$ das voluntárias dos dois grupos, sendo que, no GC, a duração foi entre 24 e 48 horas para 3 voluntárias e $(n=2)$ do GE relataram durar mais de 48 horas.

A prevalência de maiores queixas no GC foi de diarreia $(n=3)$, cefaleia $(n=4)$, cansaço $(n=3)$ e dor mamária $(n=2)$, enquanto no $G E$ os sintomas mais frequentes foram diarreia $(n=3)$, cefaleia $(n=4)$, cansaço $(n=1)$ e $(n=3)$. De acordo com o que foi avaliado no questionário, tais sintomas trazem algumas alterações associadas, como: alterações no sono, sendo no $G C(n=2)$ e $G E(n=1)$; relatos de diminuição do apetite $(n=1)$ no $G C$ e no $G E(n=2)$. Um total de $(n=4)$ do $G C$ e $(n=2)$ do GE relatou que permanece na cama por longos períodos, e $(n=3)$ em ambos os grupos relacionaram queixas como diminuição da concentração na aula e interferência no estudo e na realização das atividades de casa. Ainda, durante esse período, $(n=3)$ e $(n=4)$ do $\mathrm{GC}$ e $\mathrm{GE}$, respectivamente, sentem-se ansiosas ou deprimidas.

A frequência na aula nos últimos seis meses foi interrompida no $G C$ e no GE um dia por $(n=1)$ e de dois a três dias $(n=1)$. 0 analgésico foi o recurso utilizado por $(n=4)$ no $G C$, e uma voluntária $(n=2)$ do GE faz uso de compressas, recursoque todas citaram usar por iniciativa própria. Quando questionadas se consideram ter informações suficientes sobre menstruação, todas de ambos os grupos responderam que sim e que essas informações foram obtidas por familiares ou por médicos e outros profissionais de saúde.

A partir do questionário de Cefaleia, foram obtidos os seguintes resultados: todas as participantes de ambos os grupos já sentiram cefaleia e classificam a intensidade como forte; uma $(n=3)$ do GC e no GE costumam sentir na região frontal; no $\mathrm{GC},(n=3)$ descreveram a cefaleia como uma dor do tipo pulsátil e $(n=2)$ do GE disseram ter dor do tipo pressão. Todas as voluntárias do GC associaram a cefaleia ao estresse, enquanto no GE apenas ( $n=2)$; uma ( $n=4)$ no $G C$ e $(n=3)$ no $G E$ disseram ter cefaleia no período pré-menstrual, sendo que $(n=3)$ do GE relataram que as dores são sintomáticas no período menstrual. No GC, todas relataram que a cefaleia costuma durar horas e, no $G E_{,}(n=2)$ dura até dias. Todas as participantes relataram que a dor atrapalha a realização de atividades.

A média dos valores obtidos pela Escala Visual Analógica de Dor (EVA) antes da aplicação das técnicas do GC foi de 2,25 ( $\pm 0,5)$ e, no GE, de 2,75 $( \pm 0,5)$. Após a intervenção, a média de dor do GC foi de $1,00( \pm 0,0)$ e, no GE, de $1,25( \pm 0,5)$, sendo essas médias obtidas no teste t pareado (Tabela 2).

De acordo com a frequência relativa de cada grupo, pode-se observar que, no GC, houve alívio apenas da dor em cólica e, no GE, além da dor em baixo-ventre, houve melhora associada à cefaleia e à dor na região lombossacral (Tabela 3). Segundo o follow up, a dor em cólica reduziu após a aplicação das técnicas em ambos os grupos da mesma forma, sendo $(n=2)$ leve e $(n=2)$ com intensidade moderada. A cefaleia apresentou menor intensidade no $G E$, sendo reduzida em $(n=2)$ para dor leve, enquanto no GC a dor permaneceu moderada em todas as voluntárias. A Mobilização Uterina associada à Terapia Craniossacral resultou na redução da dor na região lombossacral em uma $(n=3)$ das voluntárias com intensidade leve e, no $G C$, a dor permaneceu moderada em $(n=4)$. A dor em membros inferiores teve melhor resultado no $G C$, com redução para leve de $(n=3)$, enquanto no GE permaneceu com intensidade moderada.

\section{Discussão}

$\infty \times \infty \times \infty \times \infty \times \infty \times \infty \times \infty \times \infty \times \infty \times \infty \times \infty \times \infty \times \infty$

Raiva, ansiedade, dificuldade de concentração, falta de interesse em atividades do trabalho e de casa, além de sintomas físicos (seios sensíveis, dor de cabeça, dores musculares e ganho de peso), caracterizam-se como sintomas da Tensão Pré-Menstrual e interferem diretamente na vida da 
mulher, prejudicando sua eficiência nos estudos e no relacionamento com colegas e familiares e tornando as semanas que precedem a menstruação mais difíceis. No presente estudo, além da queixa de dismenorreia, houve o diagnóstico da síndrome pré-menstrual em seis (75\%) das voluntárias. Segundo o estudo de Rodrigues e Oliveira ${ }^{18}$, que realizaram uma pesquisa com 101 mulheres com idade entre 15 e 40 anos, 82,4\% das participantes eram portadoras da síndrome pré-menstrual. Silva et al. ${ }^{19}$ realizaram uma pesquisa com $n$ amostral de 1.096 mulheres que apresentavam a síndrome pré-menstrual e obtiveram prevalência da irritabilidade em $71,4 \%$ das participantes, desconforto em $70,1 \%$ e cefaleia em $56,9 \%$. No presente estudo, $100 \%$ das voluntárias relataram irritabilidade, e ainda associaram a esse sintoma o desconforto em baixo-ventre e a cefaleia com intensidades entre moderada e severa.

As principais queixas relacionadas à dismenorreia primária descritas na amostra foram diarreia, cefaleia e dor mamária, trazendo como principais dificuldades a sensação de ansiedade, permanência na cama por longos períodos, diminuição da concentração nas aulas e interferência no estudo e na realização de trabalhos em casa. Rodrigues et al. (2011) realizaram uma pesquisa em que a média de idade foi de 19,4 anos, com uma amostra composta por 274 participantes.Observaram que $172(62,8 \%)$ possuíam dismenorreia, e os sintomas mais frequentes eram cefaleia, irritabilidade e diarreia. Foram reportadas limitações como ansiedade, concentração diminuída durante as aulas, permanência na cama por longos períodos, interferência no relacionamento com amigos/colegas e interferência no estudo e na realização de trabaIhos de casa, resultados semelhantesaos achados deste estudo.

Miziara et al. ${ }^{21}$, com uma amostra de 100 muIheres que se queixavam de cefaleia menstrual, puderam observar que $60,5 \%$ delas relataram intensidade severa da dor no primeiro dia e, para $56,3 \%$ destas, a dor persistia como severa no segundo dia. A qualidade da dor foi latejante para $66 \%$ e descrita como pressão para $31 \%$. Neste estudo, $87,5 \%$ das voluntárias relataram ter cefaleia no período pré-menstrual e $37,5 \%$ no período menstrual.
A Mobilização Visceral, de acordo com Jean Pierre Barral, é um conjunto de técnicas que têm como objetivo diagnosticar e tratar as disfunções viscerais. Entre elas, destaca-se a Mobilização Uterina, que tem como intuito restaurar e melhorar a circulação local, o posicionamento e o movimento dos órgãos, reduzindo, assim, as aderências e meIhorando a atividade funcional do útero e, consequentemente, do ciclo menstrual ${ }^{16}$. A Mobilização Uterina utilizada no presente estudo demonstrou uma melhora da dor em baixo-ventre (cólica menstrual), pois restabelece sua mobilidade, aumenta 0 fluxo sanguíneo e, consequentemente, a oxigenação, reduz aderências e promove efeito relaxante, porém sua utilização ainda é pouco descrita na literatura.

O principal objetivo da Terapia Craniossacral, desenvolvida por William G. Sutherland, consiste em equilibrar a flutuação do líquido cefalorraquidiano através da aplicação manual com toque muito gentil de padrões cranianos, que tornam possível para o organismo liberar as tensões que se acumularam nos tecidos, promovendo um profundo relaxamento e a sensação de bem-estar geral ${ }^{17}$. No $G E$, em que foram associadas as técnicas de Terapia Craniossacral e Mobilização Uterina, houve uma redução da dor após as suas aplicações. A dor do tipo cólica menstrual com aplicação da Mobilização Uterina (GC) teve uma melhora maior, porém no $G E$, em que foi realizada a associação das duas técnicas, houve um maior alívio associado em relação ao GC. O sistema craniossacral é constituído pelas membranas e pelo fluido cerebroespinhal, que envolvem e protegem o cérebro e a medula e influenciam seu bom funcionamento, estendendo-se desde os ossos do crânio, face e boca até o sacro. Qualquer restrição ou desequilíbrio pode trazer danos. A Terapia Craniossacral se baseia em toques sutis que estimulam os mecanismos naturais, proporcionando relaxamento profundo e provocando uma melhora global22, sendo então responsável pelo alívio da cefaleia e dor lombossacral descritas neste estudo.

De acordo com o follow up,realizado no próximo ciclo menstrual de cada participante, as voluntárias do GE relataram uma redução da cefaleia e da dor na região lombossacral maior quando comparadas com as do GC. 


\section{Conclusão}

$\infty \times \infty \times \infty \times \infty \times \infty \times \infty \times \infty \times \infty \times \infty \times \infty \times \infty \times \infty$

De acordo com os achados deste estudo, pode-se observar que as duas técnicas foram eficazes na melhora da dismenorreia primária. A associação das duas técnicas promoveu maior alívio dos sintomas associadosda dismenorreia. Este é um estudo piloto, são necessárias novas pesquisas com amostras maiores nas quais sejam criados protocolos de atendimento fisioterapêutico utilizando a associação das duas técnicas em mais de um ciclo menstrual para se obter melhores parâmetros de tratamento.

\section{Referências}

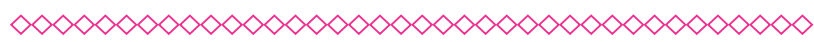

1. Bouzas I, Braga C, Leão L. Ciclo menstrual na adolescência. Adolesc Saúde. 2010;7(3):59-63.

2. Silva $A B$; Pereira AO; Silva, SP. Correlação entre as alterações posturais e a dismenorreia primária em mulheres jovens na faixa etária de 18 a 25 anos[Trabalho de Conclusão de Curso].Lins (SP): Centro Universitário Católico Salesiano;2012.

3. Teixeira ALS, Oliveira ÉCM, Dias MRC. Relação entre o nível de atividade física e a incidência da síndrome pré-menstrual. Rev. Bras. Ginec. Obst.2013;10(4):210-4.

4. Nunes JMO, Rodrigues JA, Moura MSF, Batista SRC, Coutinho SKSF, Hazime FA, et al. Prevalência de dismenorreia em universitárias e sua relação com absenteísmo escolar, exercício físico e uso de medicamentos. Rev. Bras. Promoção Saúde. 2013;26(3):381-6.

5. Sezeremeta DC, Carvalho MSS, Vrecchi MR, Marafon RGC, Crespilho LC, Pagotto JP et al. Dismenorreia: ocorrência na vida de acadêmicas da área de saúde. Ciênc. Biol. da Saúde. 2013;15(2):123-6.

6. Quintana LM, Heinz LN, Portes LA, Alfieri FM. Influência do nível de atividade física na dismenorreia. Rev. Bras. de Ativ. Física \& Saúde. 2010;15(2):101-4.
7. Gerzson LR, Padilha JF,Braz MM, Gasparetto A. Fisioterapia na dismenorreia primária: revisão de literatura. Rev Dor. São Paulo. 2014;15(4):290-5.

8. Reis AM, Souza ES, Bueno MAFA. A importância da fisioterapia no tratamento da dismenorreia primária: estudo comparativo. Rev. Cient. doCent. Unis. Católico Salesiano. 2016;15(7):502-14.

9. Oliveira RGQ, Silva JC, Almeida AF, Araújo RC, Pitangui ACR. TENS de alta e baixa frequência para dismenorreia primária: estudo preliminar. Conscientiae Saúde. 2011;11(1);149-58.

10. Bortolan HP. O conceito biodinâmico na Osteopatia. RevBrasOsteopat Ter Man.2015;6(1):6-12.

11. KLAHR, Patrícia da Silva. A influência da Terapia Craniossacral na qualidade de vida de pacientes com fibromialgia. Terapia Manual, Ijuí/ rs, p.366-373, 2011.

12. Martins MMVN. Plataforma colaborativa e cooperativa de osteopatia[Tese].Lisboa: Instituto Superior de Educação e Ciências; 2017.

13. Henz A, Wender MCO. Diagnóstico da Síndrome Pré-Menstrual: comparação de dois instrumentos - Registro Diário da Intensidade dos Problemas (DRSP) e Instrumento de Rastreamento de Sintomas Pré-Menstruais (PSST) [Dissertação].Porto Alegre: Universidade Federal do Rio Grande do Sul; 2016.

14. Prazeres LMA. Exercício físico regular, sedentarismo e características da dismenorréia e síndrome pré-menstrual [Trabalho de Conclusão de Curso].Lagarto (SE): Universidade Federal de Sergipe; 2017.

15. Lopes DCP, Führer FMC, Aguiar PMC. Cefaleia e qualidade de vida na graduação de medicina. Rev. Bras. de Neurologia e Psiquiatria. 2015;19(2):84-95.

16. Barbosa MKG. Manipulação visceral na funcionalidade dos músculos do assoalho pélvico: revisão narrativa [Trabalho de Conclusão de Curso].Campina Grande (PB): Universidade Estadual da Paraíba; 2016.

17. Kanik W, Augustyn J, Tombarkiewicz B. Adapting craniosacral therapy to treat horses. Acta Veterinaria Brno.2017;86(1):75-84. 
18. Rodrigues IC, Oliveira E. Prevalência e convivência de mulheres com síndrome prémenstrual. Arq Ciência e Saúde. 2006;13(3):61-7.

19. Silva CM, Gigante DP, Carret MLV, Fassa ACG. Estudo populacional de síndrome pré-menstrual. Rev. Saúde Públ. 2006;40(1):47-56.

20. Rodrigues AC, Gala S, Neves A, Pinto C, Meirelles C, Frutuoso C, et al. Dismenorreia em adolescentes e jovens adultas. Prevalência, factores Associados e Limitações na Vida Diária. Acta Med Port. 2011;24(2):383-92.

21. Miziara L, Bigal ME, Bordini CA, Speciali JG. Cefaléia menstrual: estudo semiológico de 100 casos. ArqNeuropsiquiatr. 2003;61(3):596-600.

22. Souza MZ de. Reflexões sobre a Osteopatia Craniana e a Terapia CranioSacral. RevBrasOsteopat Ter Man. 2012;3(2):6-7. 


\section{Anexos}

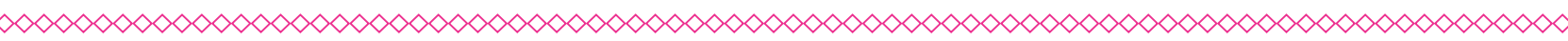

Componente: Resultados; Página 6; Linha 26.

Tabela 1 - Caracterização das voluntárias - Serra Talhada, 2019

\begin{tabular}{|c|c|c|c|c|}
\hline \multirow{3}{*}{ Variáveis } & \multicolumn{2}{|c|}{ *GRUPO } & & \\
\hline & \multicolumn{2}{|c|}{$\mathrm{GC}(\mathrm{N}=4)$} & \multicolumn{2}{|c|}{$\mathrm{GE}(\mathrm{N}=4)$} \\
\hline & Média & DP & Média & DP \\
\hline Idade & 23 & $\pm 2,16$ & 22,25 & $\pm 1,7$ \\
\hline Início da menarca & 12 & $\pm 0,816$ & 13,5 & $\pm 0,57$ \\
\hline Duração do ciclo & 25 & $\pm 4,69$ & 26,25 & $\pm 3,5$ \\
\hline Dias de menstruação & 6 & $\pm 1,14$ & 5,5 & $\pm 1,73$ \\
\hline \multicolumn{5}{|c|}{$\begin{array}{l}\text { *Percentuais obtidos com base no total de cada classificação de cada grupo; } \\
\text { GC = Grupo Controle; } \\
\text { GE= Grupo Experimental; } \\
\text { DP = Desvio Padrão. } \\
\text { N= Amostra. }\end{array}$} \\
\hline
\end{tabular}


Componente: Resultados; Página 8; Linha 4.

Tabela 2 - Intensidade da dismenorreia, quantificada pela EVA, antes e depois da aplicação das técnicas

\begin{tabular}{|c|c|c|c|}
\hline \multirow{2}{*}{ Grupo } & \multicolumn{2}{|c|}{ Dados EVA } & \multirow{2}{*}{$\mathbf{P}$} \\
\hline & Média & DP & \\
\hline GC Antes da aplicação da técnica & 2,25 & $\pm 0,5$ & \multirow{2}{*}{0,015} \\
\hline GC Após a aplicação da técnica & 1,00 & $\pm 0,0$ & \\
\hline GE Antes da aplicação das técnicas & 2,75 & $\pm 0,5$ & \multirow{2}{*}{0,014} \\
\hline GE Após a aplicação das técnicas & 1,00 & $\pm 0,5$ & \\
\hline
\end{tabular}

$\mathrm{GC}=$ Grupo Controle;

$\mathrm{GE}=$ Grupo Experimental;

$\mathrm{DP}=$ Desvio Padrão.

$p=$ nível de significância estatística para rejeição da hipótese nula. 
Componente: Resultados; Página 8; Linha 7.

Tabela 3 - Sintomas aliviados após a aplicação das técnicas de acordo com cada grupo

\begin{tabular}{ccc} 
Sintomas aliviados & $\mathrm{GC}(\mathrm{N}=4)$ & $\mathrm{GE}(\mathrm{N}=4)$ \\
Dor em cólica (baixo ventre) & $\mathrm{N}(\%)$ & $\mathrm{N}(\%)$ \\
Cefaleia & $4(100)$ & $2(50)$ \\
Dor na região lombossacral & - & $2(50)$ \\
\hline
\end{tabular}

$\mathrm{GC}=$ Grupo Controle;

$\mathrm{GE}=$ Grupo Experimental;

$\mathrm{N}=$ Amostra.

Valores expressam frequência absoluta e relativa. 